NOTAS Y DISCUSIONES

\title{
Diversidad, interpretación y reconocimiento: sobre las relaciones entre etnocentrismo y pragmatismo*
}

\author{
JOSÉ MARIA ROSALES \\ Universidad de Málaga
}

Entendido como propuesta de lectura, este trabajo trata de explorar la relevancia del pensamiento social pragmatista para el problema de la interpretación de las culturas, esto es, para el problema del conocimiento de la diferencia. El etnocentrismo plantea dicha cuestion. En su respuesta, sin embargo, quedan insuficientemente abordadas sus implicaciones prácticas, así como su articulación moral y política. En un primer apartado se presenta el papel que la interpretación como criterio metodológico para el conocimiento de la diversidad, de la diferencia, juega en las ciencias sociales. La perspectiva etnocentrista de la interpretación social es considerada en un segundo momento. Un tercer apartado describe el tratamicnto que de la interpretación y

\footnotetext{
* La redacción se ha llevado a cabo gracias al programa MEC-Fulbright durante una estancia postdoctoral en el Walt Whitman Center de la Universidad de Rutgers, en Estados Unidos. He tratado de incorporar las criticas y las sugerencias, aunque me temo que no con la habilidad que requerirfan, de Benjamin Barber, Gabriel Bello, Stephen Bronner, José M. González Garcia y José Rubio Carracedo.
}

sus implicaciones morales y políticas puede realizarse desde el pragmatismo. Por áltimo, se apunta un balance indicativo sobre el interés del pensamiento social pragmatista como alternativa epistémica y práctica al etnocentrismo. Se trata de una propuesta de lectura y en ella el hilo conductor es un cierto artificio conceptual. La referencia inmediata del debate se encuentra en la discusión entre Clifford Geertz y Richard Rorty. Por tanto, en el encuentro de dos perspectivas que probablemente experimentarán alguna evolución: la antropología interpretativa del primero y el neopragmatismo del segundo. El interés no se ha dirigido tanto a delimitar ambas posturas como a considerar algunos de los problemas planteados y a csbozar una respuesta, tentativamente razonable, desde el pensamiento social del pragmatismo clásico.

\section{I}

Durante la primera mitad de nuestro siglo la sociología interpretativa y la antropología se consolidan como ciencias sociales. Adquieren un estatuto 
epistemologico que las distingue y las independiza de las humanidades y de las ciencias naturales tras abandonar sus filiaciones historicista (que consideraba la predicción como su característica clave) y positivista (que identificaba en una misma elaboración conceptual ciencia social y explicación social). Sería el tratamiento de la interpretación como categoría metodologica y epistémica el paso decisivo hacia dicha consolidación, que reflejaría también la crisis abierta on el paradigma naturalista de la investigación social en torno al sentido del conocimiento científico. Para los autores del Círculo de Viena, desde Carnap a Reichenbach, Feigl o Hempel, la aplicación de los métodos de la física confería a las ciencias sociales una garantía de objetividad de la que carecia cualquier asimilación parcial a las Geisteswissenschaften. La observación y la experimentación constituían no sólo herramientas, sino los criterios genuinos del conocimiento científico según el enfoque positivista o empirista. Además, el empirismo tenía su contrapartida filosófica: los problemas tradicionales de la filosofía, torpemente oscurecidos por la metafísica, encontraban solución en el análisis lingüistico y a traves de la verificación de los significados. Los problemas de carácter teórico se simplificaban, en efecto, sólo que a cambio de mantener una visión naturalista o positivista del conocimiento. Pero si ya en la filosofía los últimos derroteros de la tradición analítica evidencian un agotamiento del giro lingüistico y una recuperación de la tradición pragmatista, también en las ciencias sociales el legado del positivismo es objeto de revisión. La filosofía y la investigación social comienzan a incorporar elementos de analisis hasta entonces propios de la crítica de arte, la hermenéutica textual o la his- toriografía, que cuestionan la jerarquía tradicional de métodos para el conocimiento y la primacía del sentido de la objetividad desarrollado por las ciencias experimentales. Este proceso traería consigo una creciente vinculación del conocimiento con las prácticas interpretativas. No como reducción del conocimiento a interpretacion, sino como reconocimiento del carácter reflexivo de nuestra actividad cognoscitiva. Lo cual ponía de relieve al problematizarlo el papel de interprete que asumen tanto el filósofo como el investigador social. En el segundo ejemplo, el de las ciencias sociales, la interpretacion opera como semiotica de lo incuestionado. La vida cotidiana en el caso de la sociología interpretativa y lo exótico, lo diferente, en el caso de la antropología son objeto de una reflexión que confronta los significados latentes con los significados explícitos. El resultado se traduce en una interpretación que media la distancia entre ambos niveles de sentido, sobre la cual incidirán nuevas interpretaciones que volverán a mediar la distancia significativa entre lo explícito y lo inplícito. La interpretación no viene tanto a resolver enigmas como a problematizar y esclarecer nuestro conocimiento.

En el terreno de la sociologia interpretativa y ante los problemas planteados por Weber a propósito de la comprensión de la acción social, Alfred Schutz emprende el estudio de la estructura de significados que operan en el mundo de la vida cotidiana. Sólo desde esa perspectiva, entiende Schutz, y no desde el punto de vista del observador exterior, podemos apreciar el sentido que cada sujeto da a sus propias acciones. El científico social trabaja, en efecto, sobre las interpretaciones que los individuos elaboran para comprender el mundo y para comprenderse a sí mismos, y de ahí 
que la investigación social permita, como Richard Bernstein ha destacado, una comprensión de usegundo nivel», una comprensión de la interpretación cotidiana, precientifica o de sentido común, que los individuos realizan en sus prácticas comunicativas. En su revisión del tratamiento que la fenomenología de Husserl ofrece de la intersubjetividad, Schutz integra los trabajos de George Herbert Mead sobre la emergencia del yo a través del reconocimiento interpersonal. En opinión de Schutz, a pesar de que Husserl habla de un universo de sujetos trascendentales comunicados entre sí, el conocimiento de la alteridad se lleva a cabo siempre desde la referencia de la subjetividad trascendental. Schutz corrige los excesos de la autorreferencia en Husserl mediante su analisis de la constitución intersubjetiva del mundo de la vida cotidiana. $\mathrm{Y}$ si bien, por último, el trabajo de revisión no logra perfilar un modelo de la comunicación (tarea que Habermas abordaría en su teoría de la acción comunicativa), su analítica de la interpretación ha servido de base a enfoques posteriores como la sociología fenomenológica, la sociología cognitiva, la etnometodología, la psicología descriptiva o el constructivismo social.

Para la antropología el problema de la interpretación no sólo ha propiciado frecuentes reconsideraciones de método. Ha determinado en realidad la propia orientación científica de su trabajo. Este último puede entenderse como descubrimiento de códigos de significado ocultos, como un tipo de escritura o como un modo de interpretación. Entre el estructuralismo de LéviStrauss, el textualismo etnografico de Michael Fischer o Paul Rabinow y la antropología interpretativa de Clifford Geertz media una distancia formal y de valoración de la actividad etnográfi- ca. Sin embargo, en todos encontramos un denominador común. Como James Clifford ha señalado, «en las etnografías clásicas la voz del autor se podía apreciar siempre, pero las convenciones de la presentación textual y de lectura impedían una conexión demasiado patente entre el estilo autorial y la realidad representada». La «subjetividad del autors habia de permanecer separada - continúa Clifford- de la "referencia objetiva del texto", aun a pesar de que pudiera apreciarse, sólo que en un "sentido débil", el estilo distintivo de cada autor (J. Clifford, «Partial Truths", introduccion a Writing Culture, p. 13). Dicha convención fue válida para autores como Margaret Mead o Malinowski, si bien no como regla inexorable. Pero su transgresion se haría generalizada en la generación de antropólogos que publica a partir de los años setenta. En ellos el representacionismo comienza a ser abandonado por un interés hacia la dimen. sión autorial del texto etnográfico.

Éste fue el denominador común, que la obra de Lévi-Strauss anticipa, aunque sin llegar a incorporar plenamente los elementos del giro interpretativo en las ciencias sociales. El relato de LéviStrauss, particularmente Tristes Tropiques, su autobiografia intelectual, posee ya, como Geertz ha destacado recientcmente, carácter autorreferencial, Lévi-Strauss dirige la atención del lector no sólo al mensaje, sino tambien, y de un modo incisivo, hacia la propia narrativa etnográfica. Dicha forma de textualismo no deja, sin embargo, espacio para la interpretación. Será justo en este punto donde se separen los trabajos (y los experimentos de escritura) de Rabinow o Fischer, entre otros, así como la antropología interpretativa de Geertz. Para estos autores el relato etnográfico es reconocido como trabajo de interpretacion. "El estudio interpre- 
tativo de la culturan, escribe Geertz, "representa un intento de abordar la diversidad de los modos en que los seres humanos construyen sus vidas en el acto de vivirlas", y por ello mismo ilumina la cultura del intérprete desde la perspectiva de la diferencia. Nos invita a wvernos a nosotros mismos entre los otros, como un ejemplo local de las formas que la vida humana ha tomado localmentes (C. Geertz, Local Knowledge, p. 16).

\section{II}

Si el giro interpretativo da razón de la independencia epistemológica y de método que adquieren las ciencias sociales, va a permitirnos además seguir una significativa revision posterior. Tiene que ver con lo que podriamos llamar el conocimiento de la diferencia, es decir, el conocimiento o la interpretación de las culturas. Pero no de la cultura occidental, cuyo análisis quedaba reservado a la sociologia, sino de las culturas no-occidentales, que eran estudiadas por la antropologfa. Tradicionalmente el conocimiento de la diferencia remitía a culturas no-occidentales. La investigación social operaba sobre el supuesto, en realidad contrastable, de que las sociedades occidentales participaban de una misma herencia cultural. Hoy en día el supuesto sigue siendo válido, de no ser por la salvedad de que dicha herencia se ha hecho más diversa.

A raíz de los procesos de descolonización se transforman las pautas etnográficas. "La antropología», comenta James Clifford, "ya no habla con autoridad automática por otros, definidos como incapaces de hablar por sí mismos ("primitivos", "analfabetos", "sin historia")» (J. Clifford, "Partial Truths», p. 10). Por otra parte, el final del colonialismo obliga a diseñar una política de la diversidad para integrar de un modo no violento las identidades colectivas, historias e intereses hasta entonces reprimidos. Desde los años cincuenta ésta ha sido la tónica en las transiciones a la democracia. Pero en las últimas dos décadas la política de la diversidad ha dejado de ser un parámetro distintivo de países del Tercer Mundo para pasar a convertirse en un fenómeno global. Las migraciones internacionales masivas, la acentuación de los problemas interétnicos, el rebrote de demandas nacionalistas y los problemas del multiculturalismo confirman una nueva situacion. Las sociedades occidentales son ahora bastante menos homogeneas. Su identidad colectiva común es una identidad apenas sobre mínimos. La diferencia es ya parte de lo que tradicionalmente no había sido diferente.

Como resultado, el sentido de algunas coordenadas de la investigación social es puesto en cuestión. Las correlaciones entre identidad y diferencia, objetividad y subjetividad o relativismo y universalidad son reexaminadas a la luz del cambio en las pautas ctnograficas. La dimensión interpretativa, que refería el carácter polisémico del conocimiento en las ciencias sociales, había puesto de relieve tras la revisión su dimensión práctica. Quedaba patente que la investigación social se elabora sobre presupuestos, reconocidos o no, cuestionados o no, tanto epistémicos como morales. Las interpretaciones tienen, en efecto, algún referente moral, pero por su carácter reflexivo llegan a afectar también a nuestro modo de entender la moralidad (al definir perspectivas diferentes) y a nuestras propias prácticas morales. La interpretación de la diferencia no sólo implica una modificación en el conocimiento. Tiene además consecuencias para la acción. Sobre estas premisas la dife- 
rencia, la alteridad, acaba por ser entendida como problema práctico. En su análisis moral y político puede apreciarse el abanica de problemas que de algún modo el etnocentrismo simplifica y a los que la tradición pragmatista puede, también de algún modo, responder.

Interpretar implica decodificar las referencias simbolicas, los significados de una acción, un texto, una obra de arte o una cultura. Implica hacer explícito el contenido de dichas manifestaciones o significantes, que se recrean en un lenguaje distinto al que los produjo. La decodificación constituye, por tanto, una traduccion. Desde una perspectiva etrocentrista la traducción opera como una asimilación de significados desde la cultura interpretada hacia la cultura del intérprete. En el proceso la decodificación descontextualiza los significados originales bajo el supuesto de que sólo es cognoscible lo que podemos reproducir en nuestro propio lenguaje. Lo que aparece como intraducible no es lógicamente interpretable. Su sentido permanece oculto a menos que se encuentre alguna equivalencia con significados de la cultura del intérprete. En ese momento los significados son filtrados desde las referencias simbólicas de la cultura receptora. Como puede desprenderse, la actitud etnocéntrica postula una inconmensurabilidad entre los universos simbólicos de culturas diferentes. De ahí que en principio refleje la actitud interpretativa que podríamos considerar natural: conocemos solo al traducir a nuestro lenguaje. A lo cual el etnocentrismo añade que en la traducción evaluamos desde los critcrios cpistémicos y morales de la cultura receptora. Pretender conocer una cultura diferente desde $s u$ propia perspectiva resultaría inviable epistemológicamente (encontraríamos siempre por resolver el problema de la traducción) y distorsionante en términos morales (afrontaríamos el riesgo de perder a desdibujar nuestras propias referencias morales). ${ }^{1}$

El etnocentrismo considera inviable toda comunicación que no suponga una asimilación interpretativa (mantener que en la interpretación de las culturas funcionan pautas de conocimiento universales sería un modo de encubrir la propia actitud etnocéntrica). Las pautas de conocimiento simplemente no pueden trascenderse. Pero esta legitimación epistémica de carácter relativista se continúa en una legitimación moral de signo anti-universalista. El etnocentrismo se sitúa así entre alternativas extremas: o la comunicación intercultural no es posible o el conocimiento viable ha de ser etnocentrico; o las pautas morales de culturas diferentes son inconmensurables o son reductibles en una interpretación etnocéntrica. La elección entre cxtremos se evitaría de estar formulada la problemática en otros términos.

El pragmatismo puede proveer algunas razoncs para pensar, en primer lugar, que una recepción etnocéntrica de la diversidad no agota el conocimiento de la diferencia. La hermenétutica de Gadamer se acercaría a esta postura al considerar que comprendemos el mundo desde nuestra propia perspectiva, si bien ello no nos habría de llevar a mantener el supuesto (etnocéntrico) de que cualquier otra comprensión "convergen en la nuestra propia. ${ }^{2} \mathrm{Y}$ en segundo lugar, puede proveer razones para pensar que el pluralismo de opciones morales se potencia, en lugar de reducirse, mediante su intercomunicación. El etnocentrismo, a pesar de su alegato de la diferencia, llega sólo a esbozar un enfoque insuficiente y cuestionablc de la interpretación social. La ctnografía termina por ser una cuestión de cómo filtrar pautas culturales, 
mientras que la comunicacion intercultural se presenta desde una visión relativista fundada en la ausencia de jerarquización moral entre las culturas. Este último punto es compartido por una visión pluralista, que, en cambio, sí defendería la posibilidad de la intercomunicación. El etnocentrismo aborda de esta forma el problema de la diferencia sin considerar su relevancia práctica. La reducción o asimilación de la diferencia cultural produce de hecho una distorsión cognoscitiva.

\section{III}

Recuperar la dimensión práctica de la interpretación de la diferencia trae consigo recuperar la dimensión práctica del conocimiento. No sólo en los términos de la continuidad entre conocimiento y acción, sino también desde la consideración del conocimiento como un modo de acción. Nuestro conocimiento e interpretación de la diferencia constituyen una actividad de carácter práctico: tanto en el sentido de incidir sobre nuestra comprensión de la diversidad cultural, como en el sentido, que William James señalara, de que hemos de actuar (que de hecho actuamos) con ese conocimiento. Por su parte, el carácter práctico del conocimiento mide de algún modo (no absoluto) su dimensión cognoscitiva. «La verdad", escribiria James, "se hace en el curso de la experiencian. El pragmaticismo de Peirce, el pragmatismo de James o el reconstructivismo de Dewey señalan la estrecha vinculación que se genera entre nuestros problemas prácticos y nuestros problemas cognoscitivos, y por tanto entre la teoría social y la teoria epistemologica.

El pragmatismo estableció un diálogo con su época. Al término de la guera civil en 1865 y durante las décadas siguientes se desarrolla en Estados
Unidos un periodo de reconstrucción nacional, Uno de sus objetivos básicos fue integrar la diversidad étnica y el pluralismo político en un orden legítimo. En el marco del debate social el pragmatismo elabora un proyecto de revitalización de la vida pública, de reconstrucción de la sociedad y sus instituciones en las coordenadas de un pluralismo democrático. El contexto social y el debate filosófico de la época no permiten hacer una comparación fácil con el presente. El multiculturalismo, la convivencia interétnica y el problema de los nacionalismos poseen hoy connotaciones apenas incipientes durante el periodo de producción de la primera generación de pragmatistas (hasta principios de los años cincuenta). En concreto, su internacionalización, así como su comprensión como política educativa en el caso del multiculturalismo, la recepción constitucional de los derechos colectivos en el caso de la multietnicidad, o el impacto de los nacionalismos en las transformaciones recientes de los estados nacionales. Sin embargo, su enfoque de método cobra interés en el marco actual de dichos problemas por la conjunción, o mejor, la dialéctica entre los momentos experiencial y reflexivo del conocimiento, por la consideración de la praxis en el trabajo de la ciencia social. Ello había implicado una revisión de la teoría epistemológica moderna que encontramos de nuevo en el debate filosofico actual.

Mantenfa Peirce que rel principio social está intrínsecamente enraizado en la logica», es decir, que el conocimiento posee carácter social, que no se descubre, sino que se construye socialmente. El pensamiento se dirige a producir, a fijar una creencia, a establecer una regla, teórica y práctica, que nos oriente en la acción y nos permita continuar con la tarea del conocimiento. 
La creencia establece una "regla de accibn», un "hábito", que por su parte, como Dewey advierte, habrá de ser provisional. Hay una elaboración personal, reflexiva, pero hay finalmente una contrastación social: «la creencia se fija en la comunidad». Pero Peirce va aún más lejos. La concepción de la realidad supone, en su opinión, la de una comunidad de conocimiento. En ella nuestras interpretaciones son validadas o refutadas. Por su remisión a la comunidad el conocimiento muestra su índole social. La imagen de la comunidad de investigadores avanzada por Peirce, o de la comunidad de interpretes avanzada por Royce, conjuga los momentos de contextualización y universalización del conocimiento: éste es revisado públicamente, recibe una validación contextual, pero al mismo tiempo, por su proyección social, el conocimiento, y por tanto también la interpretación, trascienden el ámbito de la mera subjetividad. Son construcciones intersubjetivas.

Por su parte, la intersubjetividad nos permite pensar la falibilidad del conocimiento. Las teorias -apunta James- no resuelven enigmas. Son instrumentos para la investigación. La teoría y la práctica configuran un continuo dialéctico. Sobre los pasos de Peirce formula Dewey su crítica de la epistemología cartesiana. El dualismo entre teoría y práctica, entre pensamiento y acción, desvincula la investigación científica del ámbito de la opinión, del conocimiento falible, para confinarlo a la búsqueda de esencias. Frente al esencialismo la epistemología pragmatista reintroduce la investigación científica en el ámbito de la praxis. Reconocer, en suma, la falibilidad del conocimiento, su validacion convencional, significa (con expresión de John Dewey) abandonar su función de "búsqueda de la certeza" y asumir su papel como "acción exploratoria", como reducción, aunque no eliminacion, de la incertidumbre.

Podemos seguir desde aquí la continuidad argumental entre el falibilismo del conocimiento y el carácter reconstructivo y reformista de la acción social. La epistemología nos conduce a la teoría social pragmatista. En la transición dos imágenes de fondo, la idea de comunidad y la idea de pluralismo, operan como hilo conductor. La comunidad de investigación o de interpretación, donde se conjugan los momentos de la contextualización y la universalización del conocimiento, puede ser, consecuentemente, una comunidad de interpretaciones, una comunidad también de culturas, donde la comunicación se lleve a cabo sobre el reconocimiento de la diferencia. De igual modo, sí el conocimiento de la diversidad nos sitúa en la vía del pluralismo epistemológico, desde él podemos contemplar la posibilidad de articular som cialmente un pluralismo de opciones morales y politicas. Comunidad y pluralismo no constituyen una sintesis natural. Su imbricación, en cambio, es el resultado de una elaboración argumentativa que refleja, en fin, las distintas modulaciones que los autores aportan a la teoría social pragmatista.

El lenguaje es la base y la clave es la praxis. Peirce recupera los trabajos de Aristóteles y Kant sobre el juicio, sobre la comunidad de pensamiento que se establece mediante la comunicación. Afirmar que «mi lenguaje es la suma total de mi yon significa para Peirce no sólo que comprendemos la realidad (y en cierto modo la creamos) con nuestro lenguaje, sino que además definimos con él, como advertiria Wittgenstein, los límites de nuestro mundo. El lenguaje, la comunicación, nos individualiza al socializarnos. La tesis del "hombre-signo" (que explica la indivi- 
dualización por el lenguaje) permite a Peirce elaborar la imagen de la comunidad de investigadores en los términos de una comunidad de comunicación. En opinión de Mead, que profundiza aquí la línea de trabajo señalada por Peirce, la comunicación actualiza una simultaneidad de perspectivas $y$ justamente por eso hace que sus referencias reciprocas se modifiquen. Por el intercambio intersubjetivo (la reflexión, también, no es sino otra forma de diálogo) los individuos actualizan sut propio lenguaje y lo modifican. Perciben la alteridad. Al establecer un espacio común de significados la comunicación transforma las referencias del entendimiento y trasciende los limites de la subjetividad. El lenguaje posibilita este paso hacia un marco simbólico universalizante. En el encuentro de perspectivas los individuos comparten un proceso de experiencia. Atribuyen de este modo significados comunes a los símbolos, a los gestos comunicativos que integran lo que Mead denomina un universo de discurso.

En sus escritos sobre teoría de los signos Peirce asume como premisa bá. sica la capacidad universalizante del lenguaje. En el acto de la reflexión, o del juicio como apuntara Kant, se produce un cierto desdoblamiento del yo, una flexión del sujeto sobre sí mismo. En estc sentido Peirce entiende que "una persona no es un individuo de un modo absoluton. Pero la naturalcza social del yo se experimenta propiamente, como Peirce reconoce, en el acto de la comunicación. El encuentro con los otros, la apertura hacia lo que Mead llama el «otro generalizado", socializa e individualiza. El reconocimiento de la alteridad es así el reconocimicnto de la identidad. Mead concibe el proceso de apertura intersubjetiva como una dinámica de continuos ajustes socializadores. El equilibrio de la comunicacion, o de la vida comunitaria, se recompone en sucesivos momentos. En la comunidad asignamos nuestras pautas de comunicación. Pero también nuestras pautas de socializacion, de aprendizaje, de autoridad, de la vida pública en definitiva. Mediante su teoría social Mead está tratando de decir que la vida comunitaria depende de la acción libre de los individuos. Es tanto un proceso de transmisión de pautas sociales heredadas, como de su reelaboración interpersonal y pública.

Gradualmente la perspectiva pragmatista articula la epistemología con la teoría social y con la teoría política. La construcción social del conocimiento, el ajuste socializador en las relaciones interpersonales, la naturaleza reconstructiva de la acción social o cl carácter reformador de la acción política configuran su lógica intema. Los trabajos de Dewey constituyen la sintesis más integradora. Si Peirce anticipa su crítica a la epistemologia cartesiana y Mead su reconstructivismo social, James y Royce avanzan respectivamente (más en un sentido conceptual que cronológico) su visión del pluralismo y su idea de comunidad. El pragmatismo de James se elabora sobre la imagen de un universo abierto. Su incompletud implica la posibilidad del cambio, de la exploración. Un universo pluralista muestra sque nada real cs simple de un modo absoluto, que cada fragmento de experiencia es un multum in parvo pluralmente relacionadon, que cada relación nos remite a nuevas relaciones. Un universo pluralista no describe por tanto un ciclo cerrado. El pragmatismo nos introduce en su complejidad. Por otra parte, experimentada la posibilidad del cambio, el individuo pragmatista, como George Cotkin señala, "se convierte, por definición, en un reformador". A propósito de su tratamiento de la religión el pragmatismo -observa $\mathrm{Ja-}$ 
mes- no opta por una visión optimista ni pesimista de la salvación del mundo. Antes bien, se identifica en realidad con una visión "meliorista" o liberadora. La última palabra queda en manos del individuo. Royce comparte el espíritu reformador del pragmatismo. Coincide con Kant al entender que la lealtad a la ley moral (que no es sino autolegislación) caracteriza a la vida moral. Es también la lealtad a un proyecto de vida social, pero sobre todo la lealtad de los individuos entre sí, la que permite materializar la idea de comunidad. Cómo se concilien intereses individuales e intereses colectivos no depende de una formula definitiva. La vida comunitaria es experimentación de los proyectos que resultan de la comunicación de proyectos individuales. La comunicación, en efecto, trae consigo una manifestación pública de las opciones individuales, genera el conocimiento de las diferencias. La lealtad a la vida comunal nos remite siempre a una gradación de perspectivas, de entornos comunitarios: desde la unidad social más elemental hacia la comunidad local o hacia otras formas complejas de vida social. La comunidad no es una mera síntesis de perspectivas individuales, es un proceso continuo de ajustes socializadores, y las perspectivas, como ha destacado Sandra Rosenthal, "pueden ser frecuentemente inconmensurablesw. La comunidad es de hecho un término plural. Son distintas comunidades, integradas, coexistentes, enfrentadas, interconectadas, donde los individuos se socializan. Royce entiende que las lealtades, si bien diferentes, no tienen por qué ser contradictorias entre sí. El sentido del cosmopolitismo, en su opinión, se enraíza en lo que llama «provincialismon, la lealtad hacia la comunidad que provee la identidad colectiva básica de cada individuo.

Cuando Royce pasa a hablar de la "esperanza de la gran comunidad" en su argumentación se produce un salto cualitativo. Desde esta nueva perspectiva el modelo de comunidad pluralista puede proveer alguna orientación para un orden internacional: el concierto entre las naciones puede funcionar como comunidad de comunidades. No un nuevo tipo de entidad, sino un nuevo tipo de relación, basada en el reconocimiento mutuo, así como en la adopción común de criterios de cooperación $y, l o$ que es más importante, de criterios de no-agresión o de seguridad mutua. No sorprende que Royce publique The Hope of the Great Community en 1916, «en un momento en que las cuestiones de la gran guerra están aún por decidirse». En 1927 aparecería The Public and Its Problems de Dewey, donde este autor retoma y profundiza la argumentación de Royce. En la introducción a su edición de 1946 Dewey comenta que una de las lecciones de la segunda guerra mundial ha sido el descrédito en que cac la idea de aislacionismo, que durante las primeras décadas del siglo había presidiclo las relaciones internacionales. La esperanza de la gran comunidad sc traduce en el escrito de Dewey en la «búsqueda» de la gran comunidad, que refleja, por su parte, la articulación de la vida comunitaria como vida pública en las distintas sociedades. De ahí que la búsqueda de la comunidad sea previamente una búsqueda, una construcción de la esfera pública. Se trata de la actividad cívica propia. La formación de las sociedades complejas había provocado un «eclipse de la vida pública». Los individuos experimentan una separación de los espacios comunes de acción cívica. $Y$ éstos son precisamente resultado de prácticas comunicativas, de las prâcticas participativas de los individuos en la sociedad, Reconstruirlas requiere un proceso de aprendizaje, formar lo que 
Dewey llama una "ciudadanía inteligenten, en referencia a que la recuperación de lo público pasa por una educación en la participación (en no menor medida que en la reflexión crítica) como método para reactivar democraticamente las instituciones sociales y politicas.

\section{IV}

"Ningún agregado de acción colectiva constituye de por sí una comunidad." Para cllo, la acción colectiva, entiende Dewey, ha de tener una orientación social, mientras que, por su parte, la acción social comunitaria ha de descansar en una experiencia de comunicación interpersonal. Retomamos aquí la cuestión del reconocimiento mutuo, que distingue a una comunidad pluralista de un colectivo orgánico y que nos muestra el modo en que el pragmatismo entiende la diversidad: como pluralismo, como comunidad pluralista. La imagen recoge la línea argumental del pragmatismo en su tratamiento de la epistemología, Ia teoría social y la teoría política. Justamente desde esta referencia podemos retomar; a su vez, la pregunta inicial sobre el conocimiento de la diferencia. Determinados aspectos que la interpretación etnocéntrica de las culturas subestima pueden ser abordados por el pensamiento social pragmatista.

Al considerar la dimensión práctica del conocimiento y la interpretación el pragmatismo pone de relieve la continutidad dialéctica entre conocimiento $y$ acción. Será en el terreno de la praxis donde la investigación social pueda ser entendida como exploración y no como descubrimiento de esencias. A partir de ahí la interpretación de la diversidad muestra sus implicaciones prácticas. El conocimiento de culturas diferentes afecta a nucstra autopercepción, relati- viza nuestra medida de la identidad colectiva. La diferencia ilumina la identidad, y a este respecto la interpretación en clave pragmatista constituye una exploración tanto en la alteridad como en la identidad, una exploración que comunica identidades colectivas, que dialogiza el conocimiento. La interpretación pragmatista no reduce la diversidad a una asimilación ni a un relativismo de perspectivas. Trata, al contrario, de comprenderla y explicarla como pluralismo. De este modo el pensamiento social pragmatista al enfocar el pluralismo como vía para el entendimiento de la diversidad puede también articularlo como construcción de un pluralismo democrático de opciones morales y políticas. Es el reconocimiento el inicio del pluralismo democrático. Orienta de hecho el sentido de una política democrática de la diversidad. Pero en la construcción del pluralismo la reflexión pragmatista habra de incorporar nuevas mediaciones (no en vano el pragmatismo habia surgido como diálogo entre tradiciones de pensamiento social): desde una crítica a los riesgos de homogeneización cultural por los excesos del universalismo hasta una crítica a la concepción neutralista del poder $y$ de las relaciones sociales en la tradición liberal. La política de la diversidad, concebida como política del reconocimiento, no entiende que una alternativa a la fragmentación social (por recordar un comentario reciente de Charles Taylor) haya de pasar por un reforzamiento del comunitarismo en tanto que unidad de diversidades. El pluralismo pragmatista no define ninguna entidad asimiladora. Propugna, en cambio, un tipo de relación encaminada a reducir asimetrías, a propiciar el entendimiento, pero no a hornogeneizar las perspectivas. Sobre el marco de los retos planteados por el multiculturalismo, la convivencia interétnica o 
el nacionalismo puede punerse a prueba la relevancia del pensamiento social pragmatista como alternativa ante el problema del conocimiento y la articulación, moral y política, de la diversidad.

\section{REFERENCIAS}

BERNSTEIN, R.J.i Beyond Objactivism and Relativism, Filadellia, University of Pennsylvania Press, 1983.

-:- The Resurgence of Pragmatism*, Social Research, 59 (1992), 813-840.

CAMPBEL, J: The Community Reconstructs, Urbana/Chicago, University of Illinois Press, 1992.

Curford, L., y Marcus, G.E. (eds.), Writing Culture, Berkeley, University of California Press, 1986.

CotkIn, G.: Willant James, pubtic Philosopher, Baltimore/Londres, The Johns Hopkins University Press, 1990.

Dewey, J.: The Later Works of John Dewey, 19251953 (ed. de J.A. Boydston), Carbondale Edwardsville, Southern Illinois University Press, 1981-1990; esp. vols. 2, 4, 11 y 17.

GeERT, C.: The Interpretation of Cultures, Nuewa York, Basic Books, 1973.

-: Local Knowledge, Nueva York, Basic Books, 1983.

- "The Uses of Diversitys, Michigan Quarterly Review, 25 (1986), 105-123.

- Works and Lives, Stanford, Stanford University Press, 1988.

HILEX, D.R., BOHMAN, J.F, y SHustermaN, R. (eds.), The Interpretive Tum, Ithaca/Londres, Cornell University Press, 1991.

JAMES, W.: The Works of Willian James (ed. de
F.H. Burkhardt), Cambridge/Londres, Haryard University Press, 1975; esp. Pragmatismt (1975) y A Pluralistic Universe (1977).

LYotaRd, J.-F:: *Histolre Universelle et Diffúrences Culturelles», Critique, 456 (1985), 559-568.

Mrad, G.H.: Mind, Self and Society (1.* ed., 1934) (ed. de C.W. Morris), Chicagollondres, The University of Chicago Press, 1962.

-: Selected Writings (ed. de A,J, Reck), Chicagollondres, The University of Chicago Press, 1964.

Peirce, C.S.: Collected Papers of Charles Sanders Peirce (ed de C. Hartshorne y P. Weiss), vol. V: Pragnatism and Pragmaticism, Cambridge, Harvard University Press, 1934.

RoRTY, R.: \&Le Cosmopolitisme sans Émancipation: En Reponse a J.F. Lyotard a, Critique, 456 (1985), 569-580.

-: OOn Ethnocentrism: A Reply to Clifford Geertz», Michigan Quarterly Review, 25 (1986), 525-534.

Rosenrmal, S.B.: Speculative Pragnatism, Amherst. The University of Massachusetts Press, 1986.

Rovee, J.; The Basic Writings of Josiah Royce (ed. J.J. McDermott), 2 vols., Chicago/Londres, The University of Chicago Press, 1969.

Sowutz, A.: Collected Papers (ed. de M. Natanson et al, 3 vols.. La Haya, M. Nijhoff, 1962-1966.

\section{NOTAS}

1. No en otro sentido se pronunciaba LéviStrauss en su discurso de 1971 ante la UNESCO. "Raza y Culturan, que recientemente ha servido a Geertz ( $($ The Uses of Adversity', pp. 106 ss.) para desatar la polémica en torno al etnocentrismo en la interpretación cultural, y cuya primera respuesta procedio de Richard Rorty. El neopragmatismo de Rorty se concilia con lo que ell llama un "etnocentrísmo moderado* (a Le Cosmopolitisme sans Emancipation", pp. 570 ss.), que si bien no comparte la apelación emancipadora de la teoría moral de Habermas ni la desconfianza militante de Lyotard hacia los proyectos universalistas de la Ilustración (cE. aHistoire Universelle et Différences Culturelles $x$ ), permite una recepción de los problemas del pluralismo y el progreso moral. No universalismo, pero st cosmopolitismo; no emancipación, pero sí reforma o progreso moral. El pragmatismo clasico, como se verá más adelante, anticipa alguros de los aspectos de un etnocentrismo moderado, pero se pronuncia, en concreto en el caso de Dewey, por un moderado universalismo moral, que se amoniza con los contenidos de un pluralismo democrático.

2. La idea de convergencia para caracterizar la interpretación etnocentrista y distinguirla de la hermenéutica pracede de David $\mathrm{C}$. Hoy en su trabajo "Is Hermeneutics Ethnocentric?", aparecido en The Interpretive Tum, pp. 155-175. 